\title{
Calcium Imaging of Neuronal Activity under Gradually Changing Odor Stimulation in Caenorhabditis elegans
}

Yuki Tanimoto ${ }^{1, \$}$ and Koutarou D. Kimura1, 2, *

\begin{abstract}
${ }^{1}$ Graduate School of Science, Osaka University, Toyonaka, Japan; ${ }^{2}$ Graduate School of Science, Nagoya City University, Nagoya, Japan; ${ }^{\$}$ Current address: Center for Brain Science, RIKEN, Wako, Japan

*For correspondence: kokimura-lab@umin.ac.jp or kokimura@nsc.nagoya-cu.ac.jp
\end{abstract}

\begin{abstract}
[Abstract] Olfactory behavior is among the most fundamental animal behaviors both in the wild and in the laboratory. To elucidate the neural mechanisms underlying olfactory behavior, it is critical to measure neural responses to odorant concentration changes resembling those that animals actually sense during olfactory behavior. However, reproducing the dynamically changing olfactory stimuli to an animal during such measurements of neural activity is technically challenging. Here, we describe technical details and protocols for odor stimulation during calcium imaging of the sensory neurons of the nematode Caenorhabditis elegans. In this system, the neuronal activity of $C$. elegans is measured using ratiometric calcium imaging during exposure to quantitatively controlled olfactory stimuli over time. Temporal changes in odor concentrations around the animal are precisely controlled according to a predesigned temporal odor gradient to reproduce a realistic odor concentration change during olfactory behavior in a behavioral arena. By monitoring neural activity in response to the realistic olfactory stimulus, it is possible to elucidate the mechanisms by which olfactory input is processed by neural activities and reflected in behavioral output.
\end{abstract}

Keywords: Behavior, Caenorhabditis elegans, Calcium imaging, Navigation, Olfaction

[Background] Olfaction is an important modality that almost all animal species use for survival and reproduction, and olfactory behaviors, such as pheromonal responses, innate/learned fear responses, and olfactory navigation, have been intensively studied (Bargmann, 2006; Jacobs, 2012; Isosaka et al., 2015; Yabuki et al., 2016). These behaviors are guided by dynamically changing sensory input. For example, approaching or withdrawing from an odor source causes gradual changes in the odor concentration sensed by an animal. Because odor concentration information is usually not delivered smoothly or consistently but rather unequally and/or with certain noise, the nervous systems of animals must process dynamically changing sensory stimuli properly using temporal differentiation and/or integration (Tanimoto and Kimura, 2019).

To elucidate the neural mechanisms underlying olfactory behavior, it is crucial to measure neural responses to gradually changing olfactory stimuli similar to what animals actually sense during the behavior. However, in most conventional studies, step-wise stimuli (i.e., the sudden presentation and/or withdrawal of an odor stimulus) have been typically used instead of realistic gradual changes in odorant concentration. This is partly because it is technically challenging to stimulate animals with a realistic 
gradual concentration change because it is difficult to: (1) measure the odor concentration actually sensed by an animal during olfactory behavior; and (2) quantitatively control the odor stimuli presented to awake behaving animals to reproduce the measured realistic gradual concentration changes. Accordingly, conventional studies have increased our understanding of only limited aspects of olfactory behavior; thus, the neural mechanisms regarding the spatio-temporal information processing of olfactory stimuli are still largely unknown.

The nematode Caenorhabditis elegans has been widely used to study olfactory behavior. C. elegans shows attractive and repulsive behaviors to odor stimuli, and has a strong advantage for neural studies because of its simple nervous system with complete connectome information (White et al., 1986; Bargmann et al., 1993; Bargmann, 2006). C. elegans is also useful for calcium imaging of neural activity because of its transparent body and the availability of various cell-specific promoters to express genetically encoded calcium indicators (GECls) (Kerr, 2006). Furthermore, recent technical advances have enabled the application of calcium imaging to freely moving $C$. elegans by using automated tracking of the animal using a motorized stage (Faumont et al., 2011; Kawano et al., 2011; Piggott et al., 2011; Zheng et al., 2012; Tanimoto et al., 2016). This method allows for the simultaneous analysis of behavioral output and neural activity, such as motor neuron or command interneuron activity. However, during calcium imaging, it has remained difficult to present a realistic gradual odorant concentration change. A widely used microfluidic flow channel delivers only up-step or down-step odorant stimuli to a physically restricted animal in a small chamber (Chronis et al., 2007). Systems that deliver gradually changing concentrations of water-soluble chemicals/odorants were recently developed (Albrecht and Bargmann, 2011; Larsch et al., 2013; Itskovits et al., 2018), but these odor delivery methods use only the aqueous phase, which differs from the widely used air phase olfactory paradigm (Bargmann, 2006). In addition, such microfluidic channels are not compatible with auto-tracking microscope systems for calcium imaging of freely moving $C$. elegans at high magnification. Therefore, it has been technically difficult to simultaneously analyze neural activity and behavior in response to naturalistic olfactory stimuli in freely moving $C$. elegans.

Here we describe the technical details and protocols of our original calcium imaging microscope system integrated with a specialized odor stimulation device. In this system, temporal changes in odor concentration around the animal are precisely controlled according to predesigned temporal odor gradients to reproduce olfactory stimuli during olfactory behavior. These temporal gradients are designed based on measured odor concentration changes sensed by the animal during actual olfactory navigation behavior in a classic Petri dish experiment (Yamazoe-Umemoto et al., 2015 and 2018). This odor stimulation device can also be combined with an auto-tracking motorized stage to apply the realistic graded odorant stimuli to freely moving $C$. elegans. By reproducing realistic odor concentration changes during the measurement of neural activity and behavior, it was possible to elucidate the neural mechanisms that underlie the dynamic aspects of olfactory processing by the nervous system, such as temporal differentiation and integration (Tanimoto et al., 2017). Our protocol for the repulsive odor 2-nonanone described below should be applicable to other odorants. 


\section{Materials and Reagents}

1. Regular $6 \mathrm{~cm}$ sterile Petri dish (Valmark, catalog number: 6-8627-02) with Nematode Growth Medium (NGM) agar (see the Recipes section and Materials and Reagents items 16-23 for ingredients and details) and a bacterial lawn of Escherichia coli strain OP50 (Caenorhabditis Genetics Center)

Note: Spread OP50 on the NGM plate and allow it to grow for $2-3$ days at $22-24{ }^{\circ} \mathrm{C}$ under $30-50 \%$ humidity. Keep the plates on a bench during the period to dry properly.

2. Micro-volume syringe, $5 \mu$, fixed needle (SGE, catalog number: 001000)

3. Microliter syringe, $50 \mu \mathrm{l}$, cemented needle (Hamilton, catalog number: 80565)

4. $25 \mathrm{ml}$ Gastight Syringe (Hamilton, catalog number: 82520)

5. Luer-lock side-hole needle, $23 \mathrm{G} \times 4 \mathrm{~cm}$ (GL Sciences, catalog number: 3008-46004)

6. Perfluoroalkoxy alkane (PFA) Teflon tube with inner and outer diameters of $0.5 \mathrm{~mm} \times 0.7 \mathrm{~mm}$ (Aram, catalog number: 1037-05)

7. PFA Teflon tube with inner and outer diameters of $1.0 \mathrm{~mm} \times 2.0 \mathrm{~mm}$ (Aram, catalog number: 1029-51)

8. Regular silicone tube with inner and outer diameters of $1.0 \mathrm{~mm} \times 2.0 \mathrm{~mm}$ (Aram, catalog number: 1018-03)

9. Polyethylene (PE) tube with inner and outer diameters of $0.5 \mathrm{~mm} \times 1.0 \mathrm{~mm}$ (Hibiki, No. 3, catalog number: 94-1161)

10. Tube fitting, $Y$ style, 1/16" barb (Warner Instruments, catalog number: 64-1571)

11. Plastic disposable syringe A8450 (Henke, Germany)

12. $34 \mathrm{~cm} \times 24 \mathrm{~cm}$ plastic case $2321 \mathrm{E}$ (Sanplatec, Japan)

13. Micro manipulator MF-1 (S Company, Japan)

14. C. elegans strain expressing GCaMP and mCherry in target neuron(s) using a cell-specific promoter cultivated at $20^{\circ} \mathrm{C}$ for 4 days on the NGM+OP50 plate

Note: We used strains KDK23009, KDK23032, KDK23034, and KDK52204 for imaging of the AWB neuron and strains KDK70034, KDK70231, KDK70239, and KDK70265 for imaging of the ASH neuron (Tanimoto et al., 2017). Other cell-specific promoters and GECls can be used in principle.

15. 2-Nonanone (Wako Pure Chemical Industries, catalog number: 132-04173)

Note: Liquid at room temperature.

16. Sodium chloride ( $\mathrm{NaCl}$ ) (Wako Pure Chemical Industries, catalog number: 191-01665)

17. Bacto peptone (BD Bacto ${ }^{\mathrm{TM}}$, catalog number: 211677 )

18. Agar (Wako Pure Chemical Industries, catalog number: 010-08725)

19. Cholesterol (Wako Pure Chemical Industries, catalog number: 034-03002)

20. EtOH (Wako Pure Chemical Industries, catalog number: 057-00456)

21. Magnesium sulfate heptahydrate $\left(\mathrm{MgSO}_{4} \cdot 7 \mathrm{H}_{2} \mathrm{O}\right)$ (Wako Pure Chemical Industries, catalog number:131-00405) 
22. Dipotassium hydrogen phosphate $\left(\mathrm{K}_{2} \mathrm{HPO}_{4}\right)$ (Wako Pure Chemical Industries, catalog number:164-04295)

23. Potassium dihydrogen phosphate $\left(\mathrm{KH}_{2} \mathrm{PO}_{4}\right)$ (Wako Pure Chemical Industries, catalog number:169-04245)

24. Levamisole hydrochloride (Wako Pure Chemical Industries, catalog number: 123-04641)

25. NGM plate (see Recipes)

26. NGM buffer (see Recipes)

27. $\mathrm{KPO}_{4}$ buffer ( $\mathrm{pH} 6.0$ ), $1 \mathrm{M}$ (see Recipes)

28. $200 \mathrm{mM}$ levamisole solution (see Recipes)

\section{Equipment}

1. Fluorescent microscope for calcium imaging

The details of the optical system for calcium imaging are shown in Figure 1. This system can be used either for a freely moving animal by automated tracking with the motorized stage or for immobilized animals under an anesthetic (levamisole). The animal is exposed to excitation light from a mercury lamp (USH-1030L, Olympus, Japan) through a BP460-495 bandpass-filter (Olympus) and a DM505HQ dichroic mirror (Olympus). This excitation light is weakened to approximately $100 \mu \mathrm{W} / \mathrm{mm}^{2}$ (measured by optical power meter HIOKI 3664+9742) with neutral density (ND) filters (Olympus) and used for both GCaMP and mCherry excitation. The fluorescence of GCaMP and mCherry is acquired using the 20x UPLFLN objective lens (Olympus) and introduced into a W-View beamsplitter (Hamamatsu, Japan). The W-View contains two dichroic mirrors (545SP and 545LP) and bandpass filters (D520/20m and 620DF35) to split the GCaMP and mCherry fluorescent images (Figure 1). These two images are simultaneously captured side-by-side on an EM-CCD camera ImagEM (Hamamatsu). The fluorescent intensities of GCaMP and mCherry must be set at a similar level; otherwise, fluorescence from the stronger channel may leak to the weaker channel. The mCherry fluorescence is stronger than the GCaMP fluorescence with our optical setup, and the use of blue excitation light, which excites mCherry less efficiently than green excitation light does, can balance the fluorescence intensities of the mCherry and GCaMP. Fluorescent images are taken at a 32.6-ms exposure time and 100-ms sampling interval with $2 \times 2$ binning. 


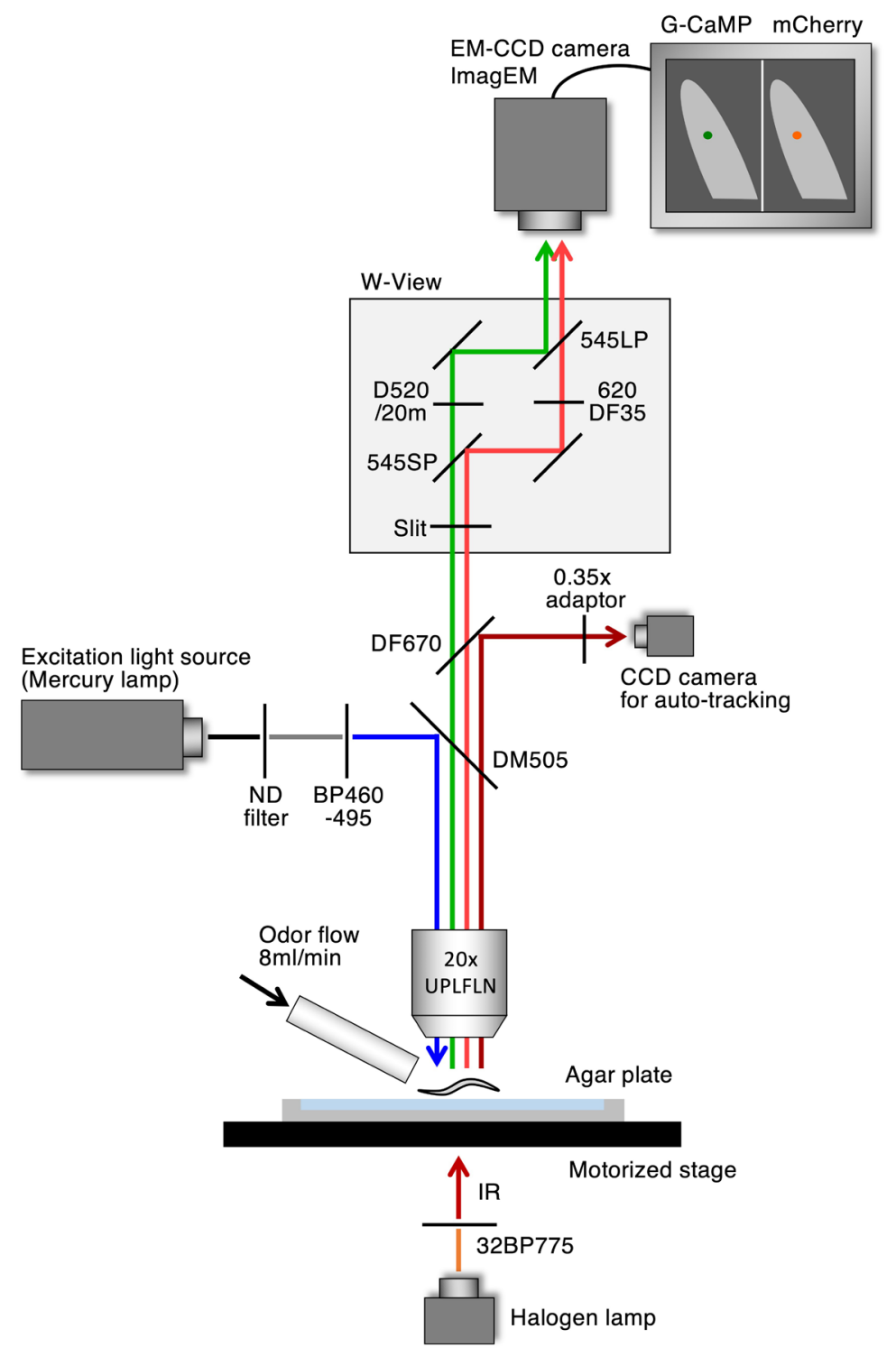

Figure 1. A schematic of the microscope system used for calcium imaging under the controlled odor stimulus. The system also possesses an auto-tracking function. The system comprises multiple bandpass filters [BP460-495 (Olympus), 32BP775 (Olympus), D520/20m (Chroma), and 620DF35 (Omega)], dichroic mirrors [DM505, 545SP, 545LP (Olympus), and DF670 (Semrock)], and neutral density (ND) filters (Olympus). The W-View enables image-splitting for dual-wavelength imaging using a single camera (Hamamatsu Photonics). The ImagEM electron-multiplying charge-coupled device (EM-CCD) camera is used to acquire fluorescent images sensitively (Hamamatsu Photonics). IR, infrared light.

2. Odor delivery system

Although this odor delivery system was initially developed as a part of an auto-tracking system for freely moving $C$. elegans, we later used it for multiple immobilized animals (Tanimoto et al., 2017; Yamazaki et al., 2019). During imaging, an animal (or multiple immobilized animals) is continuously exposed to an odor flow from a tube end placed $\sim 1 \mathrm{~mm}$ away from it. When this 
system is used with the auto-tracking system, the odor flow from the tube also continuously stimulates the animal since the animal is tracked in the center of the view field (Figure 1). Temporal changes in the 2-nonanone concentration in the odor flow are controlled by two HV-SSP01 syringe pumps (HawkVision, Japan), each holding two $25 \mathrm{ml}$ Gastight syringes (Hamilton) (Figure 2). One pump is used to infuse the 2-nonanone gas, while the other infuses air, and the infused gas and air are mixed through a custom-made microchannel (see Figure 2 and Equipment step 3). The two pumps are programmed to deliver a constant $8 \mathrm{ml} / \mathrm{min}$ gas flow in total from the tube end with varying combinations of pump speed to cause the temporal changes in 2-nonanone concentration. For example, when the pump speed for 2-nonanone is changed from $2 \mathrm{ml} / \mathrm{min}$ to $5 \mathrm{ml} / \mathrm{min}$, for air it is changed from $6 \mathrm{ml} / \mathrm{min}$ to $3 \mathrm{ml} / \mathrm{min}$ during the same period. With the $8 \mathrm{ml} / \mathrm{min}$ odor flow, the animal's entire body is exposed to an essentially uniform odor flow without significant stagnation, diffusion, or turbulence. We found that the 8 $\mathrm{ml} / \mathrm{min}$ air flow rate did not affect the animal's behavior, although stronger flow rates caused aversive responses of the animal. Flow rates slower than $8 \mathrm{ml} / \mathrm{min}$ reduce the temporal precision of the temporal odor gradient when measured with a semiconductor odor sensor (see Equipment steps 5-6). The experimental room, particularly around the microscope, must be kept windless during the experiment. The behavior of the odor flow can be visualized by filling fog produced by Wizard Stick (Zero Toys, USA) instead of the odor gas (see Tanimoto et al., 2017, Video 3).

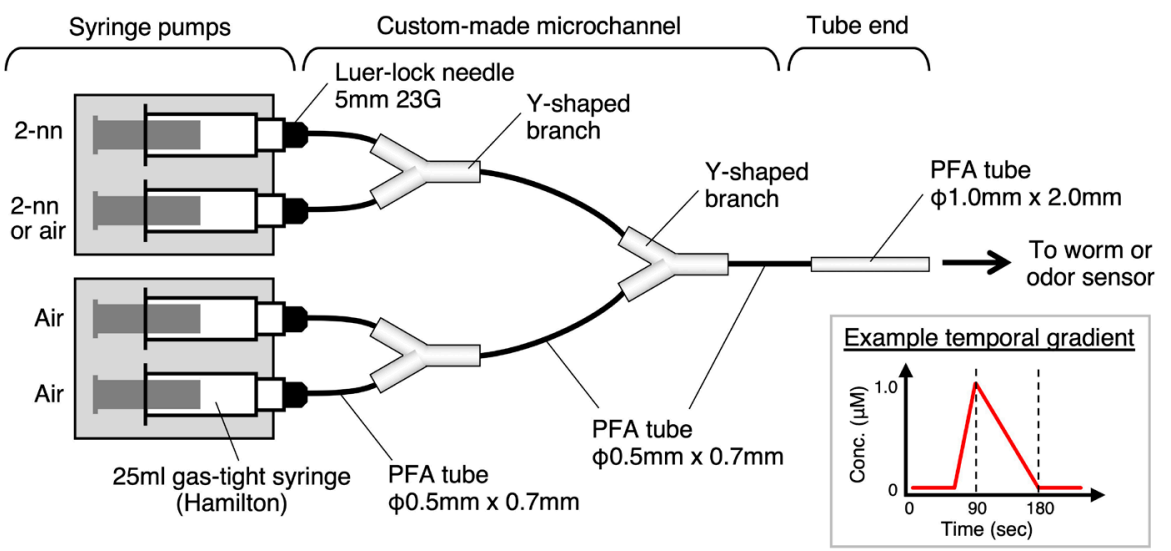

Figure 2. A schematic of the odor delivery system and custom-made microchannel for odor delivery. PFA tube $\varnothing 0.5 \mathrm{~mm} \times 0.7 \mathrm{~mm}$ indicates a tube with an inner diameter of $0.5 \mathrm{~mm}$ and an outer diameter of $0.7 \mathrm{~mm}$. The graph shows a sample temporal odor gradient.

3. Custom-made microchannel for odor delivery

We fabricated a custom microchannel for odor delivery. Because 2-nonanone, the odorant we used here, is highly adsorptive, we chose glass, stainless steel, and PFA Teflon to fabricate the microchannel since they are less adsorptive to 2-nonanone. Other kinds of plastics or silicones are highly adsorptive to 2-nonanone, and we avoided using them for the channel. The following 
steps comprise a sample fabrication procedure (Figure 2):

a. Cut four Luer-lock side-hole needles $23 \mathrm{G} \times 4 \mathrm{~cm}(3008-46004$, GL Sciences) at $5 \mathrm{~mm}$ from the basement of the needle (Figures 2, 3A).

Note: Do not chop off the needle since it may crush and close the needle hole. Rather, pinch the needle with nipper blades at the position to cut and then scratch the outer surface of the needle by rotating it. After scratching the needle several times, you can easily tear it off.

A
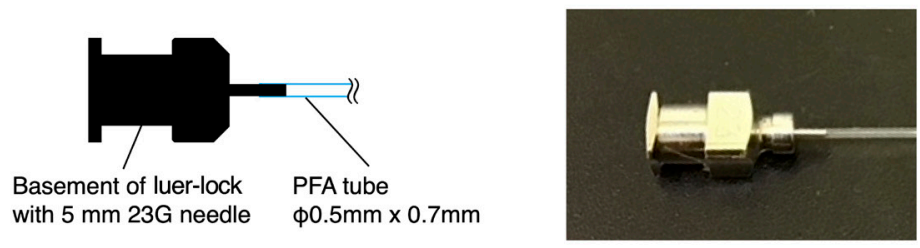

B

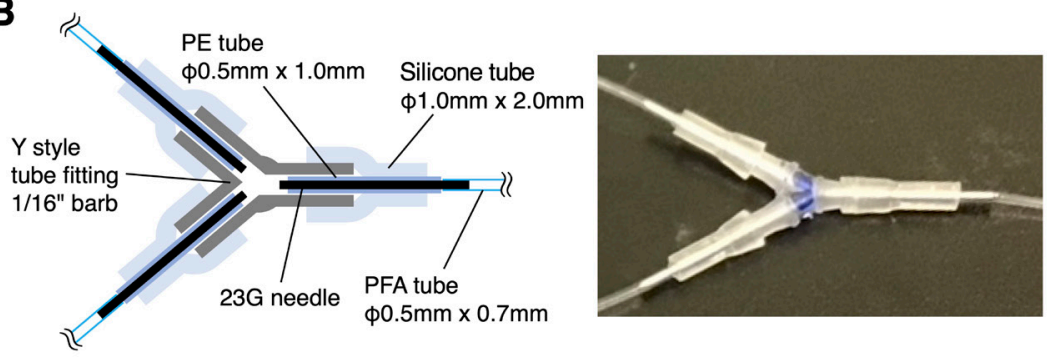

C

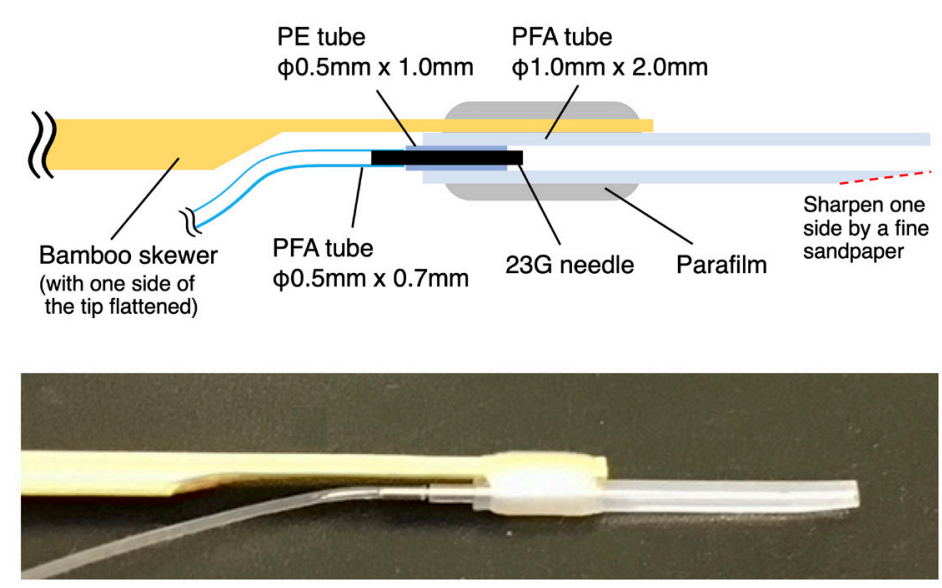

Figure 3. Detailed structures of the custom-made microchannel. A. A schematic and photograph of the Luer-lock basement of a $23 \mathrm{G}$ needle attached to a $\varnothing 0.5 \mathrm{~mm} \times 0.7 \mathrm{~mm}$ PFA tube (left and right, respectively). B. A schematic and photograph of the detailed structure of the Y-shaped branch. C. A schematic and photograph of the detailed structure of the tube end attached to a bamboo skewer. 
b. Assemble three Y-shaped branches from the $23 \mathrm{G}$ needles cut out from the Luer-lock side-hole needle, regular silicone tubes $(1.0 \mathrm{~mm}$ inner and $2.0 \mathrm{~mm}$ outer diameters), PE tubes (0.5 mm inner and $1.0 \mathrm{~mm}$ outer diameters; Hibiki, No.3, 94-1161), and $\mathrm{Y}$ style tube fittings (1/16" barb; Warner Instruments, 64-1571) according to the drawing in Figure 3B.

c. Connect the three Y-shaped branches with seven PFA Teflon tube pieces $(0.5 \mathrm{~mm}$ inner and $0.7 \mathrm{~mm}$ outer diameters; Aram, 1037-05) and connect the four Luer-lock basements from step a above, as shown in Figure 2 "Custom-made microchannel".

Note: To ensure temporal odor gradient precision, design the channel as short as possible depending on the positions of the equipment.

d. Prepare a tube end with a $\sim 2 \mathrm{~cm}$ piece of PFA Teflon tubing (1-mm inner and 2-mm outer diameters; Aram, 1029-51) as shown in Figure 3C. Sharpen one side of the tip with fine sandpaper to deliver the odor flow to the surface of an agar plate (Figure 4, bottom right panel).

e. Connect the tube end from step $d$ to the microchannel from step c above, as shown in Figure 2 "Tube end".

f. Prepare a 15-20-cm-long bamboo skewer by flattening one side of the tip with a knife (Figure 3C, Figure 4 bottom left panel).

g. Attach the tube end to the bamboo skewer using parafilm (Figure $3 \mathrm{C}$ ), then set the bamboo skewer to a micro manipulator MF-1 (S Company) placed near the stage (Figure 4, bottom left panel). Move the tube end under the objective lens using the manipulator.

Note: We consider that 2-nonanone does not accumulate on the surface of the objective lens because it is made from glass, which is not highly adsorptive to 2-nonanone. In fact, we did not observe significant changes in neuronal and behavioral responses before and after repetitive experiments. Therefore, we assume that the effect of accumulated 2-nonanone on the surrounding materials is negligible. 


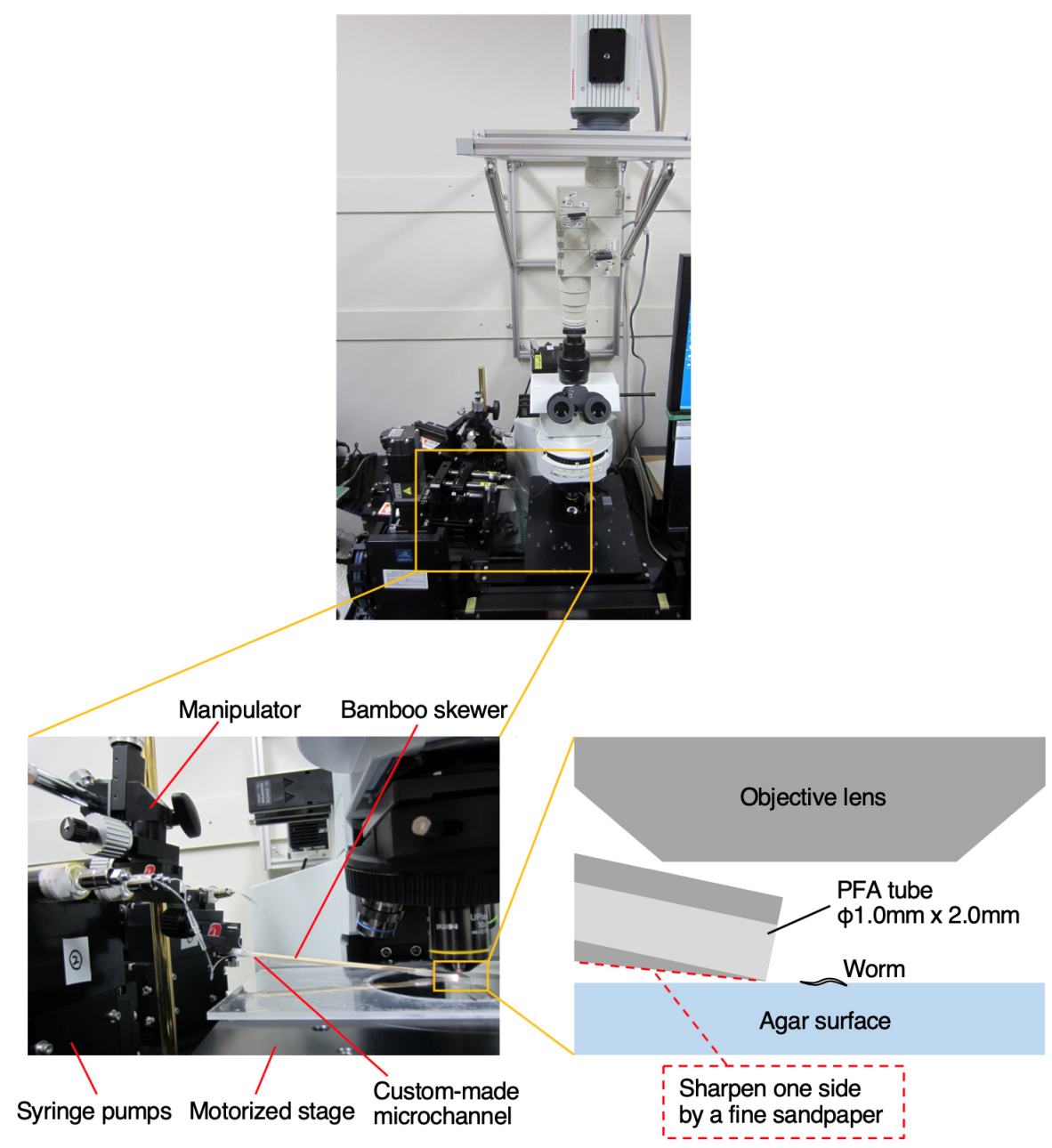

Figure 4. The microscope system and odor delivery system. A frontal view of the entire microscope system (top), arrangement of the odor delivery system and the microscope (bottom left), and a schematic drawing of the arrangement of the objective lens, agar surface, and tube end from the odor delivery system (bottom right).

4. Vaporizing tank (also see Yamazoe-Umemoto et al., 2018)

A custom-made 50-L acrylic tank is used for vaporizing and storing the 2-nonanone gas (FIS, catalog number: DT-T1) (Figure 5). The lid of this vaporizing tank is equipped with a small metal block with a groove, a heater with a temperature controller, and a fan. The odorant liquid is placed in the groove of the metal block through a liquid inlet and the metal block is warmed with the heater to facilitate volatilization of the odorant. To vaporize the 2-nonanone, put the liquid in the groove of the metal block at $50^{\circ} \mathrm{C}$. A fan stirs the gas so that the volatilized odorant is diffused equally in the tank. 


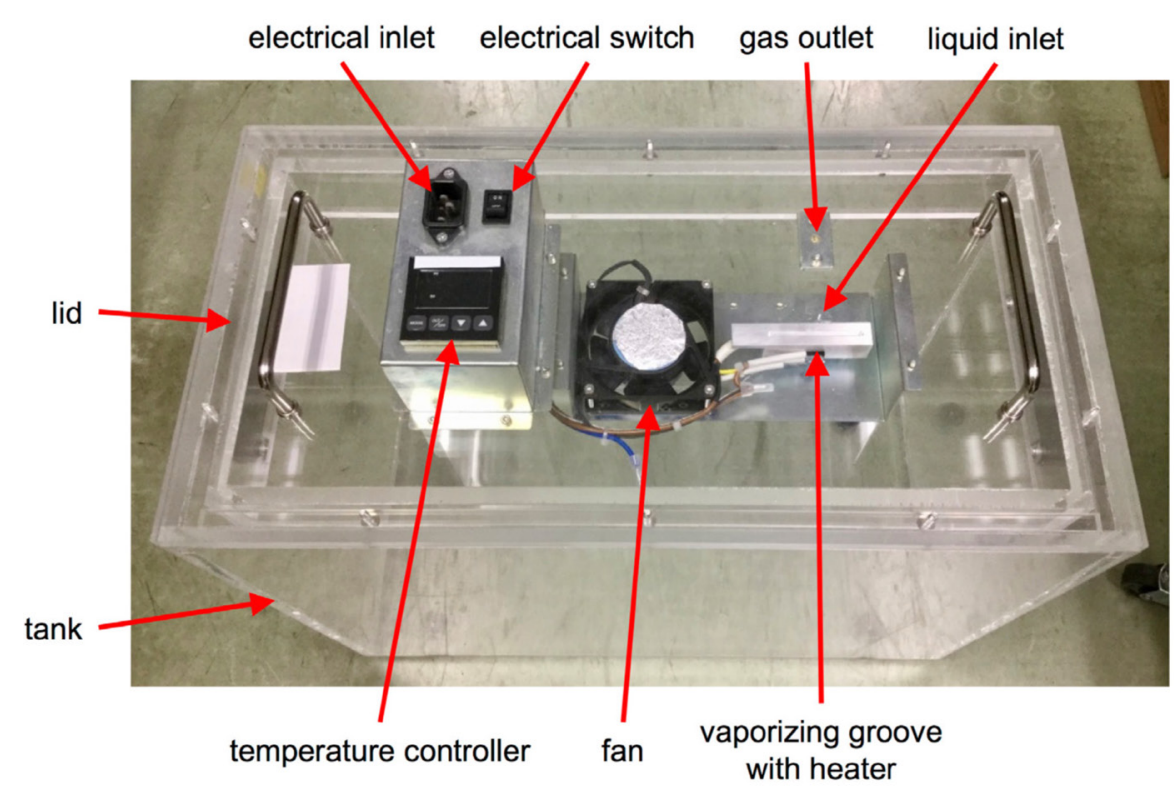

Figure 5. Vaporizing tank (adapted from Yamazoe-Umemoto et al., 2018). The tank is made of 10-mm-thick acrylic boards. The lid is equipped with an electric power supply, gas outlet, liquid inlet, vaporizing groove with a heater, fan, and temperature controller. The liquid inlet is placed just above the vaporizing groove and liquid odorant can be placed in the groove using a $5-\mu l$ micro-volume syringe (SGE) or a $50-\mu l$ microliter syringe (Hamilton).

5. Semiconductor odor sensor

The temporal concentration change of 2-nonanone is measured at the end of the tube by the semiconductor odor sensor (the same type of sensor used in a gas chromatograph SGVA-N2, FIS; Yamazoe-Umemoto et al., 2018) before and after the imaging experiments each day (Figure 6A). The voltage output from the sensor is recorded with a PC via an MAS345 digital multimeter (Mastech, Hong Kong). The sensor is calibrated on each experimental day at concentrations of $0.51,1.02,2.04$, and $4.09 \mu \mathrm{M}$ (Figure 6B). The detailed calibration and measurement procedures are shown in steps B, E, F, I in the Procedure section (Figure 6C). 
A

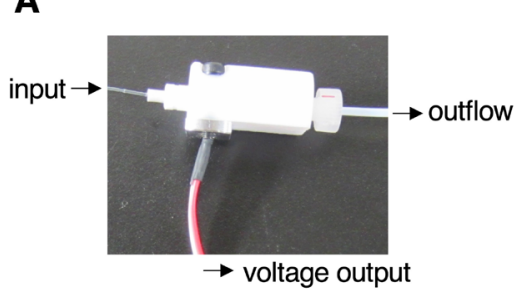

B

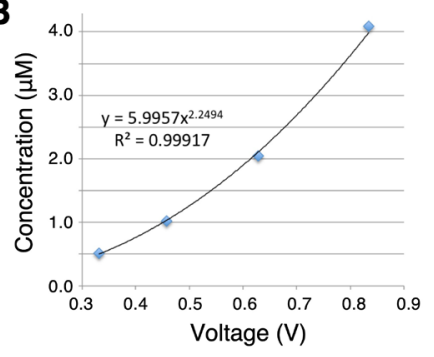

C
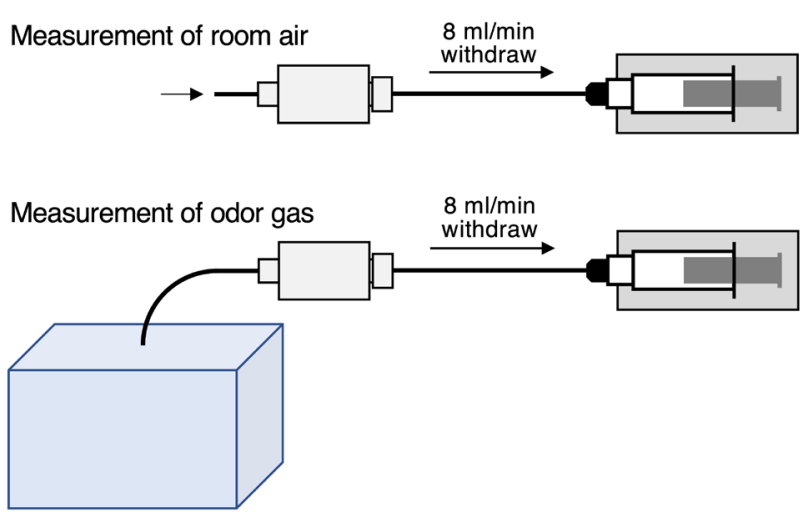

Measurement of a temporal odor gradient

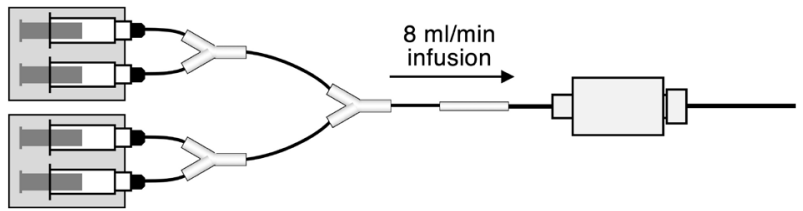

Figure 6. Application of semiconductor odor sensor. A. Semiconductor odor sensor. B. A sample calibration curve for the semiconductor odor sensor and power approximation of the voltage-concentration relationship. C. Schematics of the semiconductor odor sensor used to measure the room air (top), odor gas (middle), and temporal odor gradients (bottom).

6. Programming the temporal odor gradients

Prepare the temporal odor gradient programs prior to the imaging experiment according to the following steps:

a. Determine the number of 2-nonanone syringes (one or two) to be put on the syringe pump for 2-nonanone. The syringe pump for 2-nonanone with two 2-nonanone syringes produces $\sim 3.0 \mu \mathrm{M}$ at the maximum speed, although such high concentration is generally not required to produce the measured odor concentration change in the behavioral arena. The syringe pump for 2-nonanone with one 2-nonanone syringe and one air syringe produces $\sim 1.5 \mu \mathrm{M}$ at maximum speed.

b. Make a draft program of a temporal odor gradient with timepoint (seconds) and corresponding pump speed $(\mathrm{ml} / \mathrm{min})$ for each syringe pump for 2-nonanone and air. The sample draft program shown below is aimed to produce the temporal odor gradient shown in Figure 2 right bottom panel, with one 2-nonanone syringe and one air syringe on the 
syringe pump for 2-nonanone. Edit the draft program using a text editor and save it as a text file in the main folder of the control PC of the syringe pump HV-SSP01 (HawkVision). The intervals between the timepoints are interpolated linearly by the control program of the pump. Ensure that the changes in the speeds of the two pumps are reciprocal to keep the odor flow at a total of $8 \mathrm{ml} / \mathrm{min}$.

For the syringe pump for 2-nonanone [timepoint (s), speed ( $\mathrm{ml} / \mathrm{min})$ ]:

0,0

60,0

$90,3.0$

180,0

240,0

For the syringe pump for air (timepoint, speed):

$0,4.0$

$60,4.0$

$90,1.0$

$180,4.0$

$240,4.0$

Note: Because there are two syringes on each pump, the $4 \mathrm{ml} / \mathrm{min}$ speed produces the $8 \mathrm{ml} / \mathrm{min}$ flow rate from the pump. At $t=90(\mathrm{~s})$, one 2-nonanone ( $4 \mu \mathrm{M}$; see below) syringe and one air syringe are pushed at $3 \mathrm{ml} / \mathrm{min}$ on the syringe pump for 2-nonanone. On the syringe pump for air, two air syringes are pushed at $1 \mathrm{ml} / \mathrm{min}$. Thus, $4[\mu \mathrm{M}] \times 3[\mathrm{ml} / \mathrm{min}] \times 1$ [syringe $](3[\mathrm{ml} / \mathrm{min}] \times 2$ [syringes] $+1[\mathrm{ml} / \mathrm{min}] \times 2$ [syringes] $)=1.5 \mu \mathrm{M}$ is the expected value of the odor concentration at $t=90(\mathrm{~s})$. However, the concentration measured by the odor senor is generally lower, resulting in approximately $1 \mu \mathrm{M}$ due to adsorption of 2-nonanone to the microchannel wall.

c. According to steps B, E, F in the Procedure section (see below), use the semiconductor odor sensor to measure the actual odor concentration change of the draft program. There may be some differences between the aimed temporal odor gradient and the actual measurement results. Modify the draft program so that the differences are canceled out.

d. Repeat step c until all differences between the aimed temporal odor gradient and the actual measurement become negligible.

7. Use of open agar plates to enable air-phase olfactory stimulation of the worms Specialized agar plates must be made for this experiment. Two pieces of 2-mm-thick transparent polycarbonate plates, one with a $110 \mathrm{~mm} \times 165 \mathrm{~mm}$ rectangle shape and the other with a $110 \mathrm{~mm} \times 165 \mathrm{~mm}$ rectangle shape with an 80-mm-diameter hole, are glued with plastic bond $6225 \mathrm{~N}$ (3M, USA) to make a custom plastic dish (Figure 7). The 2-mm-deep, 80 -mm-diameter hole is filled with $10 \mathrm{ml} \mathrm{NGM}$ with $2.5 \%$ agar to create an open agar plate 
(Figures $8 \mathrm{~A}, 8 \mathrm{~B}$ ). This agar plate is especially useful for the auto-tracking experiment because the dish has no brim that can interfere with the objective lens and the bamboo skewer. Also, by filling the dish with $10 \mathrm{ml} \mathrm{NGM}$ agar, we can reduce the meniscus and increase the flatness of the agar's surface to ensure stable focusing during tracking.
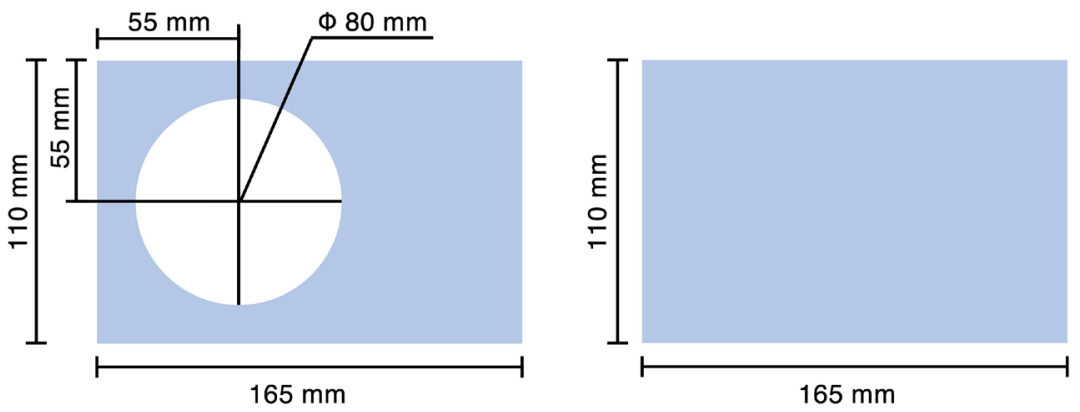

Figure 7. Open agar plates enable air-phase olfactory stimulation of the worms. Drawings of two polycarbonate plates for a custom-made plastic dish of the open agar plate.

\section{Software}

1. ImageJ software $(\mathrm{NIH})$

\section{Procedure}

A. Preparation of C. elegans strains

1. Transfer four adult animals expressing GCaMP and mCherry from the cultivation plate to a new NGM+OP50 plate using a platinum worm picker under fluorescent dissection microscopy guidance.

2. Keep the new plate at $19.5^{\circ} \mathrm{C}$ for $8 \mathrm{~h}$ and then remove the four adult animals transferred in Step A1.

3. Incubate the new plate at $19.5^{\circ} \mathrm{C}$ for $65-70 \mathrm{~h}$ until the start of the calcium imaging experiment. In these conditions, $\sim 100$ synchronized young adult animals will be grown with a few eggs laid on the plate per animal at the beginning of the experiment.

Note: Because some transgenic strains lay fewer eggs than wild-type animals, the number of such transgenic animals transferred to the new plate can be increased to obtain $\sim 100$ animals per plate. Fine temperature adjustment is required for robust 2-nonanone response, although this may not be the case for other odor stimuli.

B. Creation of 2-nonanone gas and a calibration curve for odor measurement using the vaporizing tank

1. Turn on the semiconductor odor sensor at least $30 \mathrm{~min}$ before use. 
2. Add $70 \mu \mathrm{l}$ of 2-nonanone into a $1.5-\mathrm{ml}$ regular laboratory tube.

3. Measure the baseline (i.e., room air) voltage level of the semiconductor odor sensor by withdrawing the room air to the sensor with a plastic syringe A8450 (Henke) set on the syringe pump at $8 \mathrm{ml} / \mathrm{min}$ for $450 \mathrm{~s}$ (Figure $6 \mathrm{C}$, top panel).

4. Make $6.13 \mu \mathrm{M}$ 2-nonanone gas in the vaporizing tank while acquiring a data set for a calibration curve on the experimental day by the following steps.

a. Put $4.45 \mu \mathrm{l}$ of 2-nonanone from the aliquot in the groove of the heating metal block in the vaporizing tank using a $5-\mu \mathrm{l}$ micro-volume syringe (SGE). Immediately start measuring the time-course of 2-nonanone vaporization in the tank. Withdraw the gas inside the tank to the odor sensor with the same equipment as that in Step B3 at $8 \mathrm{ml} / \mathrm{min}$ and record the voltage output from the sensor for $5 \mathrm{~min}$ (Figure 6C, middle panel). Confirm that the time-course of the voltage plateaued after $4 \mathrm{~min}$ and adopt the voltage at $5 \mathrm{~min}$ for the concentration of $0.51 \mu \mathrm{M}$ (approximately $25.6 \mu \mathrm{mol}$ of 2 -nonanone added to the $50-\mathrm{L}$ tank). After taking the measurement, stop the syringe pump and discard the gas inside the plastic syringe to prepare for the next measurement.

b. Add another $4.45 \mu \mathrm{l}$ of 2-nonanone to the vaporizing tank and measure the time-course of its vaporization for another $5 \mathrm{~min}$. Adopt the voltage at $5 \mathrm{~min}$ for the concentration of 1.02 $\mu \mathrm{M}$.

c. Add $8.9 \mu \mathrm{l}$ of 2-nonanone to the vaporizing tank using a $50-\mu \mathrm{l}$ microliter syringe (Hamilton) and measure the time-course of its vaporization for $8 \mathrm{~min}$. Adopt the voltage at $8 \mathrm{~min}$ for the concentration of $2.04 \mu \mathrm{M}$.

d. Add $17.8 \mu \mathrm{l}$ of 2-nonanone to the vaporizing tank and measure the time-course of its vaporization for $13 \mathrm{~min}$. Adopt the voltage at $13 \mathrm{~min}$ for the concentration of $4.09 \mu \mathrm{M}$.

e. Add $17.8 \mu \mathrm{l}$ of 2 -nonanone to the vaporizing tank. A total of $53.3 \mu \mathrm{l}$ of 2 -nonanone is evaporated in the tank, producing $6.13 \mu \mathrm{M} 2$-nonanone gas.

5. Turn off the semiconductor odor sensor.

6. Create a calibration curve to calculate the concentration from the measured voltage output. Subtract the baseline voltage value (final timepoint from Step B3) from the measured voltage values for $0.51,1.02,2.04$, and $4.09 \mu \mathrm{M}$ (from Step B4) to obtain four data points $(x, y)=$ (voltage, concentration) for each concentration. Then, fit the four data points by the power approximation and determine parameters $a, b$ of the calibration curve $y=a x^{b}$ (Figure 6B). The calibration curve will be used to calculate concentration change $y(t)$ from the measured voltage $x(t)$ in the following experiment. Create a calibration curve each experimental day because the parameters $a$ and $b$ gradually change due to the characteristic of the semiconductor sensor.

\section{Preparation of open agar plates}

1. Prepare NGM with $2.5 \%$ agar on each day of the experiment. Dissolve the compounds (Recipe section) in a microwave oven. No need for sterilized handling.

2. Keep the medium and the custom-made polycarbonate dishes at $60^{\circ} \mathrm{C}$ for about $1 \mathrm{~h}$. 
3. Bring the medium and dishes on the bench and keep the medium on a warming magnetic stirrer to retain medium-low viscosity.

4. Quickly pour $10 \mathrm{ml}$ of the medium into the dish using a 5-ml Pipetman P5000 (Gilson) before the pre-warmed dish cools to room temperature. Prepare one agar plate for one trial planned on the experimental day. Be careful not to create any bumps or dents on the agar surface that may disturb the imaging.

5. Cover four plates on the bench with a lid of a $34 \mathrm{~cm} \times 24 \mathrm{~cm}$ plastic case $2321 \mathrm{E}$ (Sanplatec) to avoid dust and excessive dryness (Figure $8 \mathrm{~A}$ ).

6. After $15 \mathrm{~min}$, move the agar plates to the $34 \mathrm{~cm} \times 24 \mathrm{~cm}$ plastic case. Close its lid and store it at room temperature (Figure 8B).

A

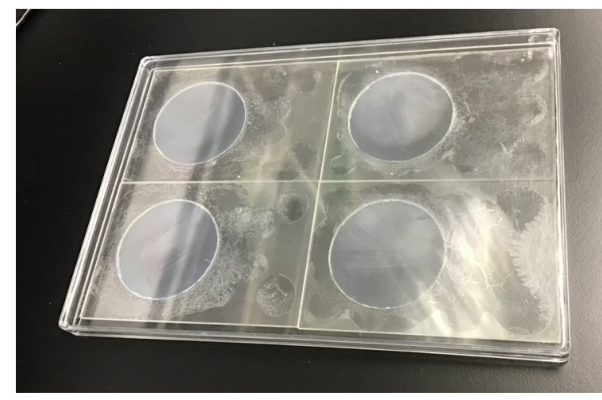

$\mathbf{B}$

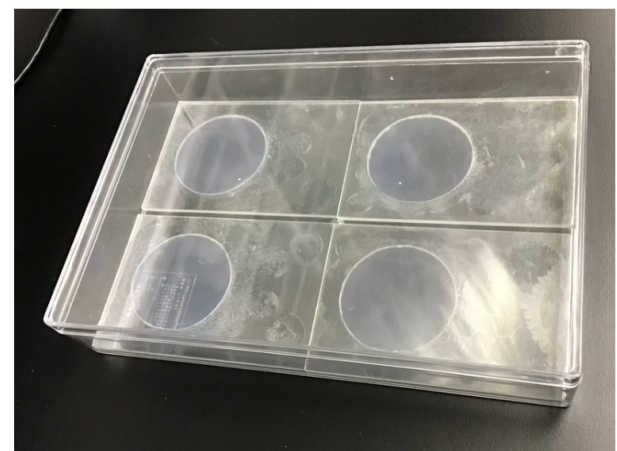

Figure 8. Creating the open agar plates. A. Four open agar plates covered by the lid of a $34 \mathrm{~cm} \times 24 \mathrm{~cm}$ plastic case 2321E (Sanplatec) on the bench. B. Four open agar plates stored in the $34 \mathrm{~cm} \times 24 \mathrm{~cm}$ plastic case with its lid closed.

D. Preparation of NGM buffer for washing the worms

1. Make $1 \mathrm{ml}$ aliquots of $\mathrm{NGM}$ buffer solution without agar before adding $\mathrm{CaCl}_{2}, \mathrm{MgSO}_{4}$, and $\mathrm{KPO}_{4}$ buffer and store them at $-25^{\circ} \mathrm{C}$ before the experiment.

2. Defrost one of the aliquots and add $\mathrm{CaCl}_{2}, \mathrm{MgSO}_{4}$ and $\mathrm{KPO}_{4}$ buffer to prepare $1 \mathrm{ml} \mathrm{NGM}$ buffer without agar on each day of the experiment. For the experiment with immobilized animals, add $25 \mu \mathrm{l}$ of $200 \mathrm{mM}$ levamisole as an anesthetic to make $1 \mathrm{ml}$ NGM buffer with $5 \mathrm{mM}$ levamisole.

E. Confirmation of odor concentration in the vaporizing tank

1. Turn on the semiconductor odor sensor again at least $30 \mathrm{~min}$ before use. 
2. Two hours after Step B4, the 2-nonanone concentration in the tank will become relatively stable; during the 2-h period, 2-nonanone adsorbs to the tank wall and its concentration decreases relatively rapidly. Measure the concentration inside the tank again using the same procedure as in Step B4 (Figure 6C, middle panel). Adopt the voltage at 2 min for the concentration in the tank.

3. Calculate the 2-nonanone concentration inside the tank using the calibration curve obtained in Step B6. The concentration is expected to decrease from the original $6.13 \mu \mathrm{M}$ to around $4 \mu \mathrm{M}$ due to the adsorption of 2-nonanone to the tank.

F. Daily confirmation of the temporal odor gradient before the experiment

1. Depending on the design of the temporal odor gradient, fill one or two 25-ml Gastight syringes (Hamilton) with the 2-nonanone gas from the vaporizing tank at least $30 \mathrm{~min}$ before use to make the 2-nonanone adsorb to the syringe. To withdraw the gas from the tank, attach a Luer-lock side-hole needle $23 \mathrm{G} \times 4 \mathrm{~cm}$ (3008-46004, GL Sciences) to the syringe and insert the needle into a rubber plug on the tank's lid. Mark each syringe "2-nonanone syringe" and exclusively use it to transport 2-nonanone. Prepare two or three "air syringes" and exclusively use them to transport room air.

2. After $30 \mathrm{~min}$, discard the gas from the 2-nonanone syringe(s) in Step F1. Fill the 2-nonanone syringe(s) with the odor gas from the vaporizing tank again and set it on a syringe pump HV-SSP01 (HawkVision) for 2-nonanone. When only one 2-nonanone syringe is used, set an air syringe filled with room air on the other holder of the syringe pump for 2-nonanone.

3. Set two air syringes filled with room air on another syringe pump HV-SSP01 for air.

4. Connect the four Luer-lock basements of the custom-made microchannel to the 2-nonanone and air syringes (Figure 2).

5. Connect the semiconductor odor sensor to the end of the microchannel (Figure 6C, bottom panel).

6. Start measurements by the odor sensor and simultaneously start moving the two syringe pumps according to the predesigned temporal gradient.

7. Repeat Steps F2-F6 twice more and calculate the temporal odor concentration changes using the calibration curve obtained in Step B6. Check if the measured temporal odor gradient is as expected and stable for the three measurements.

G. Setting the tube end position

1. Disconnect the semiconductor odor sensor from the end of the custom-made microchannel. Instead, connect the end of the microchannel to the tube end attached to the bamboo skewer and the micro manipulator MF-1 (S Company).

2. Place an agar plate on the stage and slide it under the objective lens.

3. Change the objective lens from 20x UPLFLN to 10x UPLSAPO and use the binocular eyepieces to focus on the surface of the agar plate. 
4. Move the tube end of the microchannel toward the position shown in Figure 9 using the $\mathrm{XY}$-adjusting screws of the micro manipulator.

5. Using the Z-adjusting screw of the micro manipulator, lower the tube end until it touches the surface of the agar plate.

6. Slightly raise the tube end to prevent scratching the surface of the agar plate.

7. Return the objective lens from $10 \times$ UPLSAPO to $20 \times$ UPLFLN.

\section{A}

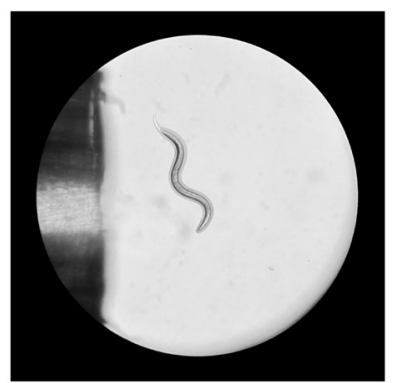

B

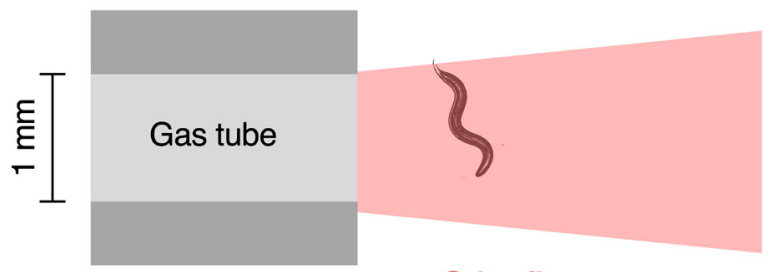

Odor flow

Figure 9. Adjusting the position of the tube end. A. Positional relationship between the tube end and a worm in the view field of the binocular eyepieces. B. A schematic of the positional relationship between the tube end and the worm.

H. Calcium imaging experiment

1. Fill the 2-nonanone syringe(s) with the odor gas from the vaporizing tank and set it on the syringe pump HV-SSP01 for 2-nonanone.

2. Fill the air syringes with room air and set them on the other syringe pump for air. When only one 2-nonanone syringe is used, set an air syringe on the syringe pump for 2-nonanone as well.

3. Connect the four Luer-lock basements of the custom-made microchannel to the 2-nonanone and air syringes (Figure 2).

4. Put two droplets of the NGM buffer (10 $\mu$ l each) on a Petri dish.

5. Using a platinum worm picker, pick up a young adult worm expressing GCaMP and mCherry from the cultivation plate prepared in Step A3 and transfer it to one of the NGM buffer droplets under fluorescent dissection microscopy. For the experiment with immobilized animals, multiple (4-10) animals can be transferred.

6. Keep the worm in the NGM buffer droplet for 1 min to wash bacterial food from the worm. 
7. Transfer the worm to the other droplet and keep it there for another 1 min for washing.

8. Transfer the worm to the center of a new open agar plate. For the experiment with immobilized animals, place multiple immobilized worms side-by-side.

9. Put the agar plate on the stage and slide it under the objective lens. Reset the position of the tube end according to Steps G3 to G7.

10. Identify the worm(s) under the microscope by moving the stage. Position the worm(s) at the center of the view field and then start the odor delivery and calcium imaging (and tracking if necessary) simultaneously.

11. After the program of the temporal odor gradient is completed, stop recording the fluorescent images. Remove the agar plate from the stage.

12. Repeat Steps $\mathrm{H} 1$ to $\mathrm{H} 11$ to increase the sample number or test other experimental conditions.

I. Daily confirmation of the temporal odor gradient after the experiment

1. Turn on the semiconductor odor sensor at least $30 \mathrm{~min}$ before use.

2. Fill the 2-nonanone syringe(s) with the odor gas from the vaporizing tank and set it on the syringe pump for 2-nonanone.

3. Fill the air syringes with the room air and set them on the other syringe pump for air. When only one 2-nonanone syringe is used, set an air syringe on the syringe pump for 2-nonanone as well.

4. Connect the four Luer-lock basements of the custom-made microchannel to the 2-nonanone and air syringes.

5. Disconnect the tube end from the end of the custom-made microchannel. Instead, connect the semiconductor odor sensor to the end of the microchannel.

6. Start the odor sensor measurements and simultaneously start moving the two syringe pumps according to the temporal odor gradient used on the experimental day.

7. Calculate the temporal odor concentration changes by the calibration curve obtained in Step B6. Check if the measured temporal odor gradient is similar to the ones obtained in Step F7.

J. Cleaning of the vaporizing tank

1. After use, open the lid of the vaporizing tank in a fume hood and keep ventilating the odor gas for at least $30 \mathrm{~min}$.

2. Wipe the inside wall of the tank with tissue paper soaked with $70 \%$ ethanol to clean up the adsorbed 2-nonanone in the tank.

3. Ventilate the tank in the fume hood with the lid open again for at least $30 \mathrm{~min}$.

4. After cleaning the tank, store it with the lid open until its next use.

\section{Data analysis}

1. Export the fluorescent images of mCherry and GCaMP as separate uncompressed 16-bit TIFF 
files (Figure 10A).

2. Track the cell body of the target neuron in the fluorescent images of mCherry. This process is required for tracking freely moving animals as well as for immobilized animals since the cell's position moves slightly even in the immobilized animals. The centroid of pixels with a brightness higher than a chosen threshold is detected as the cell position. A sub-region of the image centered on the cell position is determined and output for further analysis (Figure 10B). In the next frame, cell detection is only applied to the same area as the sub-region determined in the previous frame. It is also possible to track multiple target cells expressing mCherry individually and simultaneously using an optical flow tracking algorithm (Tanimoto et al., 2016). The result of the cell tracking obtained in the fluorescent images of mCherry can be applied to that of GCaMP by outputting the same area as the sub-region determined with mCherry from the corresponding images of GCaMP.

3. Measure the fluorescent intensities of mCherry and GCaMP using ImageJ software $(\mathrm{NIH})$. Set a circular region of interest (ROI) with an appropriate diameter on the tracked cell of the output images from step 2 manually using the ROI manager function of ImageJ (Figure 10B). Extract the area and mean brightness of the ROI using the Multi-measure function of ImageJ.

4. Measure the background intensities of mCherry and GCaMP using ImageJ. Set two circular ROIs with a five-pixel diameter on anterior and posterior regions adjacent to the tracked cell in the output images from step 2 manually (Figure 10B). Extract the mean brightness of each background $\mathrm{ROI}$ and calculate averaged mean brightness of the two background ROIs.

5. Calculate the total brightness derived from mCherry or GCaMP signals. Subtract the averaged mean brightness of the two background ROls from the mean brightness of the cell ROI, and multiply the subtracted value by the area of the cell ROI to calculate the total brightness.

6. Calculate the moving average of the total brightness values for \pm 1 frames to cancel out the noise and calculate the $\mathrm{dF} / \mathrm{F}_{0}$ of GCaMP or the ratio of the GCaMP signal over the mCherry signal.

A

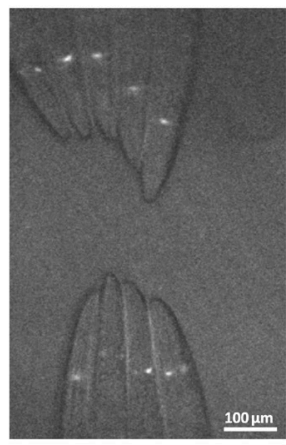

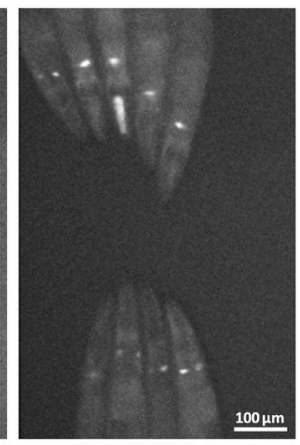

B

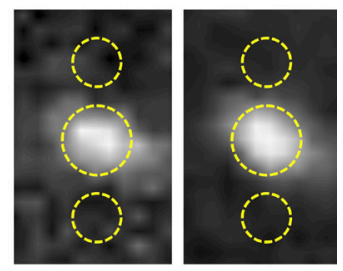

Figure 10. Data analysis of immobilized worms. A. The fluorescent images of mCherry (left) and GCaMP (right) expressed in the cell body of the ASH neurons of the immobilized animals. According to the angles of the animals, either one or two ASH neurons per animal are observed. Scale bars, $100 \mu \mathrm{m}$. B. Sub-region of the original images centered on the cell position of the 
top-right animal. mCherry (left) and GCaMP (right) images are shown. The larger and smaller circles with dashed lines indicate the cell ROI and the background ROIs, respectively.

\section{$\underline{\text { Notes }}$}

1. The temperature of the experimental room must be kept at around $21-24{ }^{\circ} \mathrm{C}$.

2. The humidity of the experimental room must be kept at $50-60 \%$ to avoid excessive dryness of the open agar plates.

3. In this protocol, we described sole use of the repulsive odor 2-nonanone, but this method should be applicable to other odorants. We tested another repellent 1-octanol using the same protocol and successfully observed an ON response in ASH neurons (unpublished observation), which was consistent with those of preceding studies (Mills et al., 2011; Zahratka et al., 2015). This protocol should be applicable to other odorants with similar physicochemical properties, such as isoamyl alcohol.

\section{Recipes}

1. NGM plate (for $1 \mathrm{~L}$ )

$970 \mathrm{ml} \mathrm{dd} \mathrm{H}_{2} \mathrm{O}$

$3 \mathrm{~g} \mathrm{NaCl}$

$2.5 \mathrm{~g} \mathrm{Bacto}^{\mathrm{TM}}$ peptone

$25 \mathrm{~g}$ agar

$1 \mathrm{ml}$ cholesterol $(5 \mathrm{mg} / \mathrm{ml} \mathrm{EtOH})$

Autoclave; wait until $50-60{ }^{\circ} \mathrm{C}$

Add the following autoclaved stock buffers:

$1 \mathrm{ml} 1 \mathrm{M} \mathrm{CaCl}_{2}$ (sterilized)

$1 \mathrm{ml} 1 \mathrm{M} \mathrm{MgSO}_{4}$ (sterilized)

$25 \mathrm{ml} 1 \mathrm{M} \mathrm{KPO}_{4}$ buffer $(\mathrm{pH}=6.0)$ (sterile)

2. NGM buffer (for $100 \mathrm{ml}$ )

$97 \mathrm{ml} \mathrm{ddH_{2 } \mathrm { O }}$

$0.3 \mathrm{~g} \mathrm{NaCl}$

$0.25 \mathrm{~g} \mathrm{Bacto}^{\mathrm{TM}}$ peptone

$0.1 \mathrm{ml}$ cholesterol $(5 \mathrm{mg} / \mathrm{ml} \mathrm{EtOH})$

Autoclave; wait until $50-60{ }^{\circ} \mathrm{C}$

Make 1-ml aliquots in 1.5-ml tubes and store them at $-25^{\circ} \mathrm{C}$

Before use, add the following buffers:

$1 \mu \mathrm{l} 1 \mathrm{M} \mathrm{CaCl}_{2}$

$1 \mu \mathrm{l} 1 \mathrm{M} \mathrm{MgSO}_{4}$

$25 \mu \mathrm{l} 1 \mathrm{M} \mathrm{KPO}_{4}$ buffer $(\mathrm{pH}=6.0)$ 
3. $\mathrm{KPO}_{4}$ buffer $(\mathrm{pH}=6.0), 1 \mathrm{M}$

$108.3 \mathrm{~g} \mathrm{KH}_{2} \mathrm{PO}_{4}$

$35.6 \mathrm{~g} \mathrm{~K}_{2} \mathrm{HPO}_{4}$

Add $\mathrm{dd}_{2} \mathrm{O}$ up to $1 \mathrm{~L}$

Autoclave

4. $200 \mathrm{mM}$ levamisole solution

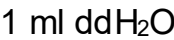

$48 \mathrm{mg}$ levamisole hydrochloride

Make $0.2 \mathrm{ml}$ aliquots in regular $1.5-\mathrm{ml}$ tubes and store them at $-25^{\circ} \mathrm{C}$

\section{Acknowledgments}

We thank Y Mori (HawkVision Inc., Japan) for providing technical support for the syringe pumps, $\mathrm{K}$ Tanaka (FIS Inc., Japan) for providing technical support for the odor delivery system and measurements, Y Ikejiri and Y. Endo for making Figure 10, and Dr. Jared Young for providing suggestions and comments about the manuscript.

Funding sources: This work was supported by a Grant-in-Aid for JSPS fellows (YT), the Osaka University Life Science Young Independent Researcher Support Program, Precursory Research for Embryonic Science and Technology from MEXT, and research grants from Mitsubishi Foundation, Shimadzu Science Foundation, and Takeda Science Foundation (KDK).

Original research paper from which this protocol was derived: Yuki Tanimoto and Akiko Yamazoe-Umemoto et al., (2017).

\section{Competing interests}

The authors declare no competing interests.

\section{References}

1. Albrecht, D. R. and Bargmann, C. I. (2011). High-content behavioral analysis of Caenorhabditis elegans in precise spatiotemporal chemical environments. Nat Methods 8(7): 599-605.

2. Bargmann, C. I., Hartwieg, E. and Horvitz, H. R. (1993). Odorant-selective genes and neurons mediate olfaction in C. elegans. Cell 74(3): 515-527.

3. Bargmann, C. I. (2006). Chemosensation in C. elegans. WormBook: 1-29.

4. Chronis, N., Zimmer, M. and Bargmann, C. I. (2007). Microfluidics for in vivo imaging of neuronal and behavioral activity in Caenorhabditis elegans. Nat Methods 4(9): 727-731.

5. Faumont, S., Rondeau, G., Thiele, T. R., Lawton, K. J., McCormick, K. E., Sottile, M., Griesbeck, O., Heckscher, E. S., Roberts, W. M., Doe, C. Q. and Lockery, S. R. (2011). An image-free opto-mechanical system for creating virtual environments and imaging neuronal 
activity in freely moving Caenorhabditis elegans. PLoS One 6(9): e24666.

6. Isosaka, T., Matsuo, T., Yamaguchi, T., Funabiki, K., Nakanishi, S., Kobayakawa, R. and Kobayakawa, K. (2015). Htr2a-Expressing Cells in the Central Amygdala Control the Hierarchy between Innate and Learned Fear. Cell 163(5): 1153-1164.

7. Jacobs, L. F. (2012). From chemotaxis to the cognitive map: the function of olfaction. Proc Natl Acad Sci U S A 109 Suppl 1: 10693-10700.

8. Itskovits, E., Ruach, R., Kazakov, A. and Zaslaver, A. (2018). Concerted pulsatile and graded neural dynamics enables efficient chemotaxis in C. elegans. Nat Commun 9(1): 2866.

9. Kawano, T., Po, M. D., Gao, S., Leung, G., Ryu, W. S. and Zhen, M. (2011). An imbalancing act: gap junctions reduce the backward motor circuit activity to bias C. elegans for forward locomotion. Neuron 72(4): 572-586.

10. Kerr, R. A. (2006). Imaging the activity of neurons and muscles. WormBook: 1-13.

11. Larsch, J., Ventimiglia, D., Bargmann, C. I. and Albrecht, D. R. (2013). High-throughput imaging of neuronal activity in Caenorhabditis elegans. Proc Natl Acad Sci U S A 110(45): E4266-4273.

12. Mills, H., Wragg, R., Hapiak, V., Castelletto, M., Zahratka, J., Harris, G., Summers, P., Korchnak, A., Law, W., Bamber, B. and Komuniecki, R. (2012). Monoamines and neuropeptides interact to inhibit aversive behaviour in Caenorhabditis elegans. EMBO J 31(3): 667-678.

13. Piggott, B. J., Liu, J., Feng, Z., Wescott, S. A. and Xu, X. Z. (2011). The neural circuits and synaptic mechanisms underlying motor initiation in C. elegans. Cell 147(4): 922-933.

14. Tanimoto, Y. and Kimura, K. D. (2019). Neuronal, mathematical, and molecular bases of perceptual decision-making in C. elegans. Neurosci Res 140: 3-13.

15. Tanimoto, Y., Yamazoe-Umemoto, A., Fujita, K., Kawazoe, Y., Miyanishi, Y., Yamazaki, S. J., Fei, X., Busch, K. E., Gengyo-Ando, K., Nakai, J., lino, Y., Iwasaki, Y., Hashimoto, K. and Kimura, K. D. (2017). Calcium dynamics regulating the timing of decision-making in C. elegans. Elife 6: e13819.

16. Tanimoto, Y., Zheng, Y. G., Fei, X., Fujie, Y., Hashimoto, K. and Kimura, K. D. (2016). In actio optophysiological analyses reveal functional diversification of dopaminergic neurons in the nematode C. elegans. Sci Rep 6: 26297.

17. White, J. G., Southgate, E., Thomson, J. N. and Brenner, S. (1986). The structure of the nervous system of the nematode Caenorhabditis elegans. Philos Trans R Soc Lond B Biol Sci 314(1165): 1-340

18. Yabuki, Y., Koide, T., Miyasaka, N., Wakisaka, N., Masuda, M., Ohkura, M., Nakai, J., Tsuge, K., Tsuchiya, S., Sugimoto, Y., and Yoshihara, Y. (2016). Olfactory receptor for prostaglandin F2a mediates male fish courtship behavior. Nat Neurosci 19(7): 897-904.

19. Yamazaki, S. J., Ohara, K., Ito, K., Kokubun, N., Kitanishi, T., Takaichi, D., Yamada, Y., Ikejiri, Y., Hiramatsu, F., Fujita, K., Tanimoto, Y., Yamazoe-Umemoto, A., Hashimoto, K., Sato, K., Yoda, K., Takahashi, A., Ishikawa, Y., Kamikouchi, A., Hiryu, S., Maekawa, T. and Kimura, K. 
D. (2019). STEFTR: A Hybrid Versatile Method for State Estimation and Feature Extraction From the Trajectory of Animal Behavior. Front Neurosci 13: 626.

20. Yamazoe-Umemoto, A., Fujita, K., lino, Y., Iwasaki, Y. and Kimura, K. D. (2015). Modulation of different behavioral components by neuropeptide and dopamine signalings in non-associative odor learning of Caenorhabditis elegans. Neurosci Res 99: 22-33.

21. Yamazoe-Umemoto, A., Iwasaki, Y. and Kimura, K. D. (2018). Measuring Spatiotemporal Dynamics of Odor Gradient for Small Animals by Gas Chromatography. Bio-protocol 8(7): e2797.

22. Zahratka, J. A., Williams, P. D., Summers, P. J., Komuniecki, R. W. and Bamber, B. A. (2015). Serotonin differentially modulates $\mathrm{Ca}^{2+}$ transients and depolarization in a $\mathrm{C}$. elegans nociceptor. J Neurophysiol 113(4): 1041-1050.

23. Zheng, M., Cao, P., Yang, J., Xu, X. Z. and Feng, Z. (2012). Calcium imaging of multiple neurons in freely behaving C. elegans. J Neurosci Methods 206(1): 78-82. 